\title{
Segmentation of Cell Clumps for Quantitative Analysis
}

\author{
Simon Li, Claudia Buehnemann, Bass Hassan and J. Alison Noble
}

\begin{abstract}
The increasing amounts of microscopy data generated in cell biology requires the development of automated tools for the quantitative analysis of images. Clumps of cells are difficult to segment due to the frequent lack of clear boundaries between cells and are often ignored, but communication between cells is an intrinsic part of the response of cells to their environment. In addition cells often show a large variation in their responses, even within a clump, and an accurate segmentation is therefore vital to prevent the unwanted averaging of measurements over multiple cells. Here we present a method for segmenting clumps of cells by using a multi-scale ridge filter to enhance unclear boundaries. A multi-phase level set method incorporating a region competition term is used to identify a boundary for each cell based on the ridge filter response.
\end{abstract}

\section{INTRODUCTION}

High-throughput screening involves the automated handling of multiple simultaneous experiments, resulting in the generation of thousands of images of cells or tissue samples. Automated analysis tools are required to process these images to detect biologically significant phenotypes which may be used to identify samples such as novel drug compounds for further characterisation, or for detecting subtle changes which can be used to elucidate the underlying biological mechanisms governing the behaviour of a cell.

The high volume of data necessitates a trade-off between the computational resources required to analyse all images versus the benefits of extracting more information from each image. As a consequence many screens rely on isolated cells to ease the segmentation procedure, which is a prerequisite for any further analysis. However, many cultured cell types have an inherent tendency to grow in clumps and exhibit cell-cell interactions which means the behaviour of a cell will change depending on the spatial distribution and responses of its neighbours.

The requirement for an accurate cell boundary increases when the behaviour of cells is highly heterogeneous. Measurements of cell parameters are typically represented in the form of summary statistics, but this discards a lot of the information concerning the distribution of a parameter. For example, the mean and variance cannot distinguish between a unimodal and a bimodal distribution of cell responses. The segmentation of a cell in itself gives important morphological information on the state of a cell, but more importantly

This research has been funded by Cancer Research UK grant C429 and EPSRC doctoral training grant EP/E501605/1.

$\mathrm{S}$. Li and J. Noble are with the Department of Engineering, University of Oxford, UK. simon.li@dtc.ox.ac.uk

C. Buehnemann and B. Hassan are with the Sir William Dunn School of Pathology, University of Oxford, UK. allows the behaviour of other intracellular proteins to be quantified on a per-cell basis.

The work presented here is the first stage in a larger project which aims to characterise the changes and heterogeneity in the response of proteins involved in the PI3K/MAPK intracellular signalling pathways. This pathway has a vital role in the regulation of cell growth, differentiation, survival and division, and has been implicated in the initiation and progression of tumours [1]. It couples external receptors in the cell membrane to the protein transcription and translation mechanisms within the cell which allow the cell to respond to its environment.

Neighbouring cells can show markedly different responses, and a poor segmentation leads to the unwanted averaging of multiple cell responses, hiding the true behaviour. For example, tumorigenesis may originate from a small number of cells which override the normal signalling responses, and the identification of these cells and their characteristic behaviours will be useful in discovering the mechanisms which lead to cancer.

\section{A. Multiregion Segmentation}

Watershed related algorithms are often used to segment images containing multiple objects, but tend to suffer from over-segmentation. Marker controlled watershed uses a marker (in our case for example a nucleus) to fix the number of objects. Coupling this to an image dependent distance term results in a Voronoi based segmentation [2]. Although fast and effective, it takes a greedy approach to assigning pixels to each cell and in practice the evolving contour is unable to back-track, which is a problem when the cells are of significantly different sizes.

Level sets are a popular method of image segmentation, and provide an implicit representation of a contour which evolves in response to a potential function derived from the image. Edge based level sets use a potential function typically derived from the image gradient to govern the evolution of a contour [3]. A constant expansion term is used to ensure the level set keeps evolving in flat areas of the image where there would otherwise be no force on the level set. Region based level sets can be more robust than edge based level sets, but they rely on a difference in intensities or some other region statistic between the foreground and background regions of an image [4].

The extension of level set methods to multiple regions requires the addition of a competition or coupling term to ensure each image region belongs to only one segmented object. Multi-phase region based level sets can be used providing neighbouring areas to be segmented exhibit a 
difference in region statistics, and the addition of a geodesic length term calculated from the image gradient to try to pick up edges delineating regions may improve performance [5].

Here we present a novel method for the segmentation of clumps of cells in the absence of clear boundaries. The nuclei are used to generate an initial segmentation using an equipartitioning procedure. This initialisation is refined using a multi-region active contour implemented using a fast level sets method which is evolved in accordance with a ridgebased potential field, which can be tuned to the particular features of interest.

\section{DATA ACQUISITION}

MCF-7 cells are a breast cancer derived cell line which tends to grow in clumps when spread out on microscope coverslips. The cells were treated with varying concentrations of IGF2, a ligand which stimulates the PI3K/MAPK intracellular signalling pathways, fixed and stained with two core markers, as shown in Figure 1. Each image is 512-x512 pixels, and was acquired on a Zeiss LSM-510 confocal microscope with a $63 \mathrm{x}$ objective, resulting in a pixel size of 280-x-280 $\mu \mathrm{m}$.

DAPI (blue) labels DNA in the nucleus of a cell. Each cell should contain one nucleus which normally appears as an elliptical object, and its shape and texture provides useful information on the age and transcriptional activity of the cell. Phalloidin (green) labels F-actin, a long filamentous polymer formed of actin protein subunits. These filaments are found throughout the cytoplasm and contribute to the structure of the cell as well as having a role in cell motility, and examination of the locations and sizes of these filaments can allow the cell's behaviour to be deduced. In particular they form a dense mesh at the cell membrane, and should therefore be ideal for labelling the outer boundary of a cell. However, in practice boundaries between cells are often poorly defined, complicating segmentation.

Other membrane specific markers are available, but later steps of the experimental protocol results in the disruption of the membrane, whereas the phalloidin-labelled Factin shows minimal disturbance. Another benefit of the phalloidin marker is that its appearance will change as the cell grows, divides or undergoes movement in response to its environment. Additional markers can be used to label other regulators and messenger proteins within the signalling pathway, allowing levels of each protein to be compared and the variation across different cells examined.

\section{MULTI-CELL SEGMENTATION}

\section{A. Initialisation}

In this work the nuclei are assumed to be already defined, and are used to create an initial segmentation by calculating an equi-partition of the cytoplasm. An adaptive threshold is used to obtain an external edge for the cytoplasm of the entire clump of cells. The cytoplasm is then partitioned by calculating a distance transform around the binary segmentation of all the nuclei, and each pixel in the cytoplasm is assigned to the closest nucleus.

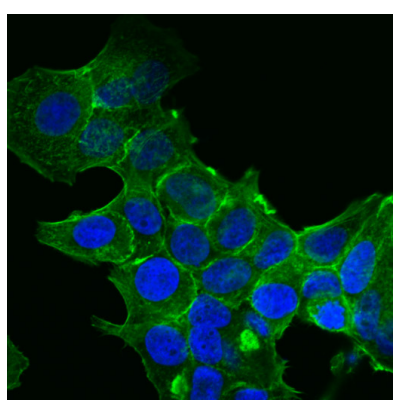

(a)

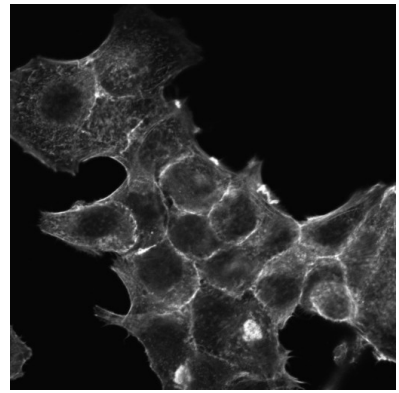

(c)

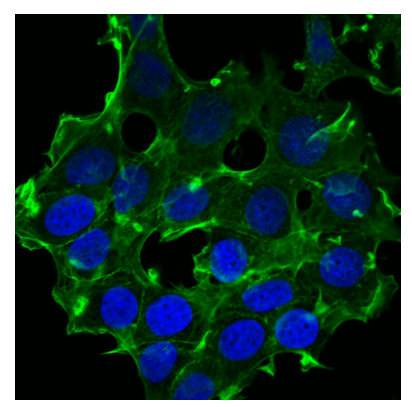

(b)

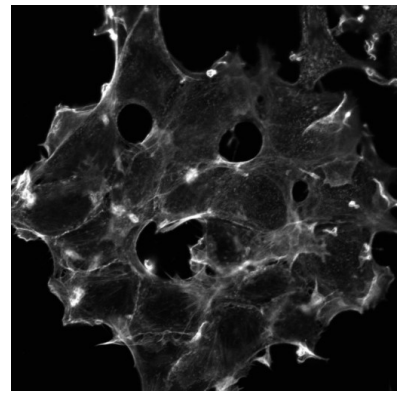

(d)
Fig. 1. Typical images of MCF-7 cells: (a),(b) DNA is stained in blue to mark the nucleus and phalloidin in green to mark the cytoskeleton; (c),(d) the phalloidin channel from (a) and (b) shown on its own. Relatively clear boundaries between the cells are visible in (a); the boundaries in (b) are far less obvious.

\section{B. Boundary Enhancement}

An edge detector [5] is non-optimal for images where the boundary is indicated by a ridge and the neighbouring regions have very similar intensities, particularly when the boundary is poorly defined. Phalloidin stained membranes have a highly variable appearance, of which the most relevant features are the bundles of F-actin filaments which can be of varying thickness.

A 2D steerable ridge filter formed from a difference-ofGaussians was therefore chosen to enhance the filaments in the phalloidin channel. A second order separable filter [6] was applied at multiple scales, with the response $f$ at each scale $i$ and orientation $\theta$ given by

$$
\begin{aligned}
f(x, y, \theta, i) & \left.=\left(-\cos ^{2} \theta+\frac{1}{3} \sin ^{2} \theta\right) \frac{\partial^{2} G_{i}}{\partial x^{2}} * I(x, y)\right) \\
+ & \left(-\frac{8}{3} \cos \theta \sin \theta\right) \frac{\partial^{2} G_{i}}{\partial x \partial y} * I(x, y) \\
& +\left(-\sin ^{2} \theta+\frac{1}{3} \cos ^{2} \theta\right) \frac{\partial^{2} G_{i}}{\partial y^{2}} * I(x, y)
\end{aligned}
$$

where $G_{i}$ is an isotropic 2D Gaussian with standard deviation $\sigma_{i}$ and $I(x, y)$ is the phalloidin stained image (Figure 2).

The filter response at any orientation $\theta$ can be calculated from just the three second-order Gaussian partial derivatives, and by setting $\frac{\partial f}{\partial \theta}=0$ the orientation giving the maximum response can be found. Each of the Gaussian derivatives are separable (meaning they can be calculated from two 1D convolutions), so this forms an efficient method of obtaining 


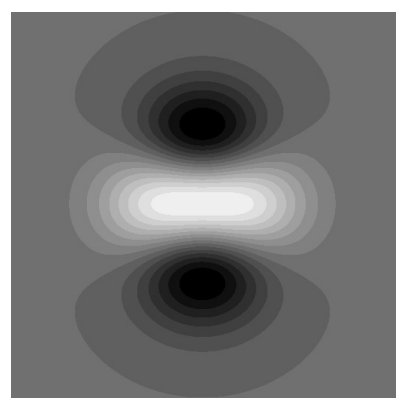

(a)

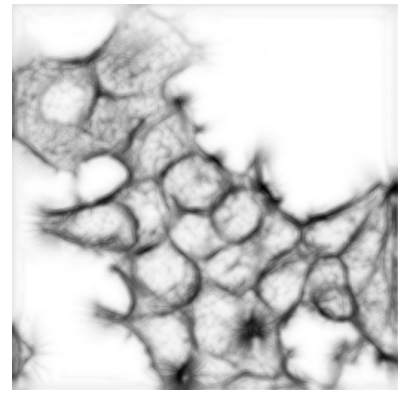

(b)

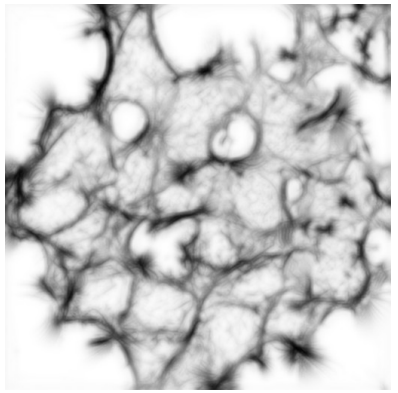

(c)
Fig. 2. (a) The ridge detector shown at orientation $\theta=0$; (b), (c) the potential fields corresponding to the images in Figure 1.

a measure of ridge strength in the image. The multiple ridge scales were chosen to detect the variable sized bundles of filaments in the images, in this case $\sigma=1,2,4,8,16$.

The filter responses were combined by taking a weighted sum

$$
F(x, y)=\sum_{i} \sigma_{i}^{2} f_{i}(x, y)
$$

where the weights account for the lower expected absolute response of the filter at larger scales, and also to place more emphasis on larger bundles since multiple separate filaments may be present. A potential function $h(x, y)$ governing the evolution of a level set was formed with a constant weighting term $\alpha$.

$$
h(x, y)=\frac{1}{1+\alpha F(x, y)}
$$

\section{Level Set Implementation}

Level sets typically require the solution of a partial differential equation, which is computationally intensive. The fast level set method developed in [7] and briefly outlined here was used as the basis for the algorithm. The speed of the algorithm is achieved by only storing two neighbouring lists of pixels for each region, one recording the pixels on the inner edge of the boundary and the other list recording those on the outer edge. At each iteration a pixel on the outer boundary may become part of the inner boundary thus causing the region to locally expand, or alternatively a pixel on the inner boundary may move to the outer boundary, resulting in the region locally contracting.

The algorithm is divided into two types of iteration:
- Speed iterations move the boundary depending on whether the speed field is positive or negative.

- Smoothing iterations replace the usual curvaturedependent regularisation with a potential field derived from the convolution of the level set representation with a Gaussian which can be used to update the contour in an analogous manner to a speed iteration.

In this initial implementation each cellular region was segmented using a separate level set. In each speed iteration the geodesic speed field proposed by [3] is used to decide in which direction a level set should evolve in response to the potential field described above.

$$
H(x, y)=h(x, y) V_{0}+\nabla h(x, y) \cdot \nabla \phi
$$

where $V_{0}$ is a constant expansion force and $\phi$ is the level set function.

If a pixel on a boundary does not overlap with another region its movement is purely governed by the sign of the speed field $H(x, y)$ at that point. However, the very weak or absent ridges would result in the level set overshooting the boundary, so in order to prevent overlap of neighbouring areas a pixel level region competition term was used. The point is assigned to the region whose speed $H(x, y)$ is the maximum of all regions competing at that point. The speeds for all regions attempting to move into a given pixel were calculated, and the region with the maximum calculated speed at that location was allowed to take that pixel with all other competing regions forced to retreat. This region competition term was also applied to the speed field calculated during the smoothing iterations.

\section{Local Minima Avoidance}

Level sets will find a local minima, and are therefore dependent on their initialisation. If a boundary exists but is some distance from the initialisation the level set may fail to find it.

These issues are handled by performing the segmentation at multiple scales. The initial image is downsampled by a factor of four, and segmented. The segmentation is then upscaled by a factor of two and the level set evolved again, though for a reduced number of iterations, and the process repeated until the original resolution is reached.

\section{RESULTS}

For the purposes of validation a set of images were taken without staining for the additional markers of the PI3K/MAPK pathways. This meant the membrane remained fully intact, and could be labelled with a reliable marker which could be used by an expert cell biologist to manually segment the images.

The manual segmentations were compared with the automated segmentations, examples of which are shown in Figure 3.

Each cell segmentation was compared using the Dice coefficient which quantified the similarity between two segmentations based on their overlaps whilst penalising any differences between them. A value of 0 indicates no overlap 

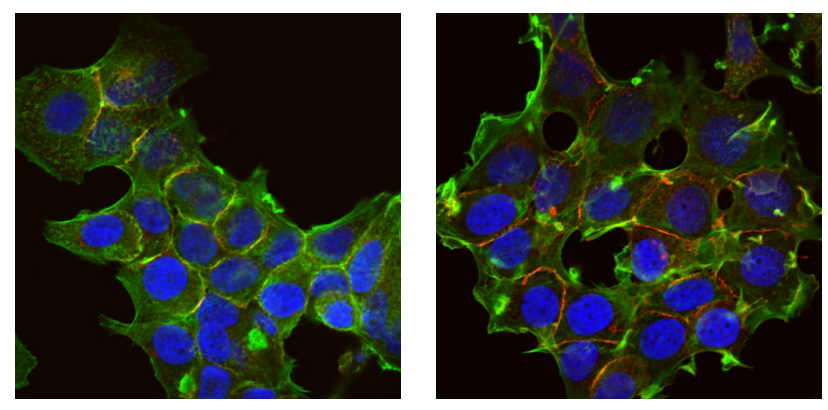

(a)

(b)

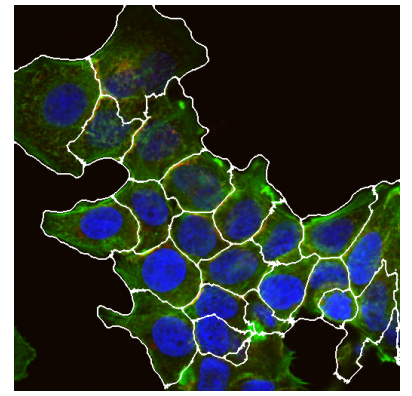

(c)

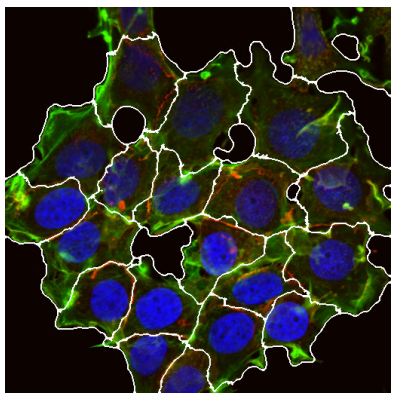

(d)
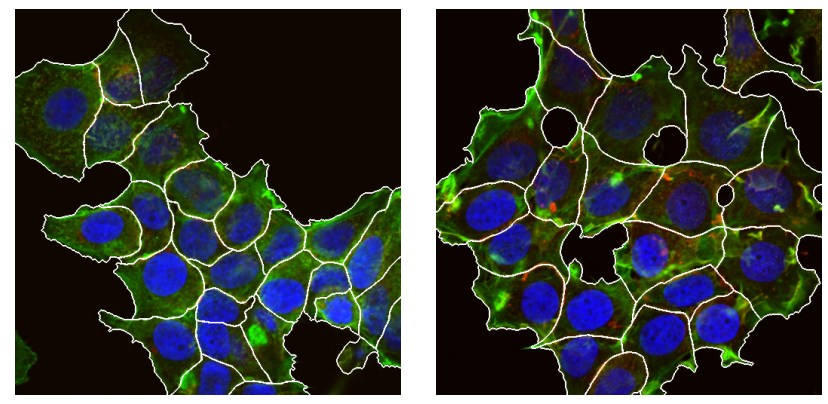

(e)

(f)

Fig. 3. Example segmentations: (a),(b) the images with the addition of a membrane marker in red, used for manual validation only; (c),(d) the automated level set segmentations; (e), (f) the manual segmentations.

between the two segmentations, a value of 1 indicates identical segmentations. The mean Dice coefficient for the cells in each image using the Voronoi method of CellProfiler [2] and the described level set method is shown in Table I.

Images 1 and 2 contain Phalloidin bundles along the cell boundaries, and the level set algorithm is able to follow these boundaries more closely due to its ability to search in both directions compared with the Voronoi approach. Images 3 and 4 contain very poor boundary information in the Phalloidin channel, and would have been very difficult to manually segment without the aid of the additional membrane marker, and the level set shows no advantage over the Voronoi method. In some cells it performs slightly worse, perhaps due

\begin{tabular}{|l|l|l|}
\hline Image & Voronoi & Level Set \\
\hline 1 & 0.85 & 0.87 \\
2 & 0.82 & 0.84 \\
\hline 3 & 0.82 & 0.81 \\
4 & 0.84 & 0.84 \\
\hline
\end{tabular}

TABLE I

DICE SIMILARITY COEFFICIENTS FOR FOUR IMAGES. THE TWO METHODS WERE COMPARED WITH MANUAL SEGMENTATIONS.

to attempting to follow ridges which do not coincide with the boundary. This suggests a method for assigning a confidence to a ridge response would be useful, though distinguishing between unclear and incorrect ridges is difficult.

\section{CONCLUSION}

A method for finding boundaries in clumps of cells in the absence of strong information has been developed. A steerable ridge filter is used to detect ridges corresponding to cell boundaries, and a fast multi-region level set is used to segment the image. Future work will include optimising the algorithm, for instance by using the four-colour theorem to reduce the computational complexity [8], and developing ways to automatically quantify the confidence in the segmentation.

This work is part of a larger project which aims to automatically search for image features which are correlated with the stimulation or inhibition of the PI3K/MAPK signalling pathways, and to relate these features back to the underlying biological mechanisms. The ultimate test will therefore be whether it can be used to obtain biologically meaningful information on the differences between cells held in different conditions, and whether the heterogeneity of cells held in the same conditions can be accurately quantified.

\section{REFERENCES}

[1] B. T. Hennessy, D. L. Smith, P. T. Ram, Y. Lu, and G. B. Mills, "Exploiting the PI3K/AKT pathway for cancer drug discovery.," Nature reviews. Drug discovery, vol. 4, no. 12, pp. 988-1004, 2005.

[2] T. R. Jones, A. Carpenter, and P. Golland, "Voronoi-based segmentation of cells on image manifolds," Computer Vision for Biomedical Image Applications, vol. 3765, p. 535, 2005.

[3] V. Caselles, R. Kimmel, and G. Sapiro, "Geodesic active contours," International Journal of Computer Vision, vol. 22, no. 1, p. 61, 1997.

[4] T. F. Chan and L. A. Vese, "Active contours without edges.," IEEE Trans. Image Process., vol. 10, no. 2, pp. 266-77, 2001.

[5] P. Yan, X. Zhou, M. Shah, and S. T. C. Wong, "Automatic segmentation of high-throughput RNAi fluorescent cellular images.," IEEE Trans. Inf. Technol. Biomed., vol. 12, no. 1, pp. 109-17, 2008.

[6] M. Jacob and M. Unser, "Design of steerable filters for feature detection using canny-like criteria.," vol. 26, no. 8, pp. 1007-19, 2004.

[7] Y. Shi and W. C. Karl, "A real-time algorithm for the approximation of level-set-based curve evolution.," IEEE Trans. Image Process., vol. 17, no. 5, pp. 645-56, 2008.

[8] S. Nath, F. Bunyak, and K. Palaniappan, "Robust tracking of migrating cells using four-color level set segmentation.," Lecture notes in computer science, vol. 4179, no. LNCS, pp. 920-932, 2006. 